\title{
THE SIGNIFICANCE OF SERUM CHOLESTEROL IN THYROID DISEASE ${ }^{1}$
}

\author{
By JOHN P. PETERS ANd EVELYN B. MAN \\ (From the Department of Internal Medicine, Yale University School of Medicine, New Haven)
}

(Received for publication July 22, 1949)

For objective evaluation of thyroid function it has long been necessary to employ indirect methods, of which the most reliable has been the measurement of basal metabolism. Although this procedure has proved a valuable aid to diagnosis, its applicability is limited by the fact that it demands cooperation on the part of the patient who is also required to be in a "basal state," an ideal condition that is not invariably attainable. Moreover, basal metabolism is not specific; variations of oxidation are not mediated through the thyroid gland alone. Other objective measures have therefore been sought. The discovery that serum cholesterol was elevated in myxedema and experimental hypothyroidism led to the clinical use of cholesterol measurements as a diagnostic aid. Although there proved to be a statistical inverse correlation between serum cholesterol and thyroid activity, in individual cases this relation as well as the correlation of cholesterol with basal metabolism were lost. It became evident that cholesterol was no more-probably less-specific than basal metabolism.

Development of precise methods for the measurement of the fraction of iodine precipitated from serum with the proteins, SPI, has provided a more nearly specific test for the functional activity of the thyroid gland. This particular fraction of the circulating iodine under ordinary circumstances appears to be composed chiefly of the thyroid hormone (1). In addition it has the advantage over other diagnostic criteria that it is not influenced by subjective factors and other features that interfere with the conduct and interpretation of measurements of basal metabolism. It has permitted the detection of disorders of thyroid function in patients who could not be tested by other objective procedures. In spite of these obvious superiorities, experience has proved that SPI is

\footnotetext{
1 Aided by grants from the U. S. Public Health Service and the Fluid Research Fund of Yale University.
}

not an absolutely specific criterion of thyroid function. Both excesses and deficiencies of SPI have been observed in patients in whom no disorder of thyroid function could be detected by clinical observation, other objective examinations or therapeutic tests.

The present paper deals with a comparison of serum lipids and SPI in patients with and without disorders of the thyroid gland. Such a comparison may aid in explaining the lack of correlation between thyroid function and SPI and cholesterol respectively and in defining more accurately the criteria for the diagnosis of disorders of thyroid function.

\section{MATERIAL AND METHODS}

Almost all of the patients were from the various services of the New Haven Hospital, the majority from the Metabolic Division. Blood for the estimation of serum lipids and SPI was drawn while the patients were in the postabsorptive state except in the case of diabetic outpatients who were taking insulin or other out-patients who lived out of town. Such exceptional patients were permitted to take a light breakfast containing no fat before coming to the hospital. This procedure does not appear to affect SPI or serum lipids and avoids danger of hypoglycemic reactions or derangement of the diabetic regime and the discomfort of prolonged activity on an empty stomach. When basal metabolism was determined, of course, the postabsorptive state was rigorously adhered to. Usually blood was drawn for lipids and SPI simultaneously; occasionally SPI and lipids were measured on successive days. In many instances serum proteins were also measured. The diagnoses of hyper- and hypothyroidism were made on the basis of history, physical examination, measurements of basal metabolism, SPI and cholesterol, and the results of therapeutic procedures.

Serum was analyzed for SPI by the method of Riggs and Man (2), for lipids by the procedures of Man and associates (3-7). Proteins were measured by a macroKjeldahl procedure. Copper or selenium and superoxal were used as catalysts up to January 21, 1946; after that mercury was employed. For fractionation the technique of Howe (8) was used through 1946, after that the technique of Milne (9) following the principle of Majoor (10). 
The concentration of the fatty acids of neutral fat in the serum was calculated by the following formula:

$\frac{10 \text { Esterified cholesterol (mg. per cent) }}{386}$

$+\frac{10[(0.80 \times 2)+0.20] \text { Lipid P (mg. per cent) }}{31}$

$=$ Fatty acids of neutral fat (mM. per liter).

It is assumed in this equation that 80 per cent of the phospholipids in serum consists of compounds (almost entirely lecithin) which contain 2 equivalents of fatty acid for each equivalent of phosphorus, and 20 per cent of compounds (sphingomyelins) in which the ratio of fatty acid to phosphorus is $1: 1$. If the proportion of sphingomyelins varied from 40 per cent to 0 , the consequent error in the conversion factor for lipid $\mathrm{P}$ would be only \pm 10 per cent.

\section{RESULTS}

The character of the clinical material investigated and the numbers and types of analyses are shown in Table I. Certain of the categories in this table require definition. Under the classification of thyroid disorders are included observations not only during active hyper- or hypothyroidism, but all observations from subjects who at some time during the course of this study had such disorders. This policy has been adopted because it permits comparison of SPI with lipids in the same individual in various phases of thyroid activity.

TABLE I

\begin{tabular}{l|r|r|r|r}
\hline \multicolumn{1}{c|}{ Condition } & $\begin{array}{r}\text { Sub- } \\
\text { jects }\end{array}$ & $\begin{array}{c}\text { Observa- } \\
\text { tions }\end{array}$ & $\begin{array}{r}\text { Com- } \\
\text { plete }\end{array}$ & Partial \\
\cline { 2 - 5 } & 125 & 222 & 85 & 137 \\
Thyroid disorders & 17 & 29 & 15 & 14 \\
? Thyroid disorders & 13 & 22 & 21 & 1 \\
Pituitary & 4 & 8 & 8 & 0 \\
Adrenal & 26 & 32 & 27 & 5 \\
Hepatic & & & & \\
$\quad$ (including steatorrhea) & 13 & 31 & 27 & 4 \\
Renal & 11 & 12 & 7 & 5 \\
Cardiac & 8 & 8 & 7 & 1 \\
Diabetic & 48 & 60 & 5 & 55 \\
Psychopathic & 72 & 80 & 38 & 42 \\
Miscellaneous & 337 & 504 & 240 & 264 \\
$\quad$ Total & & & & \\
\hline
\end{tabular}

Many of the patients under the influence of medical or surgical therapeutic measures passed one or more times between hyperthyroidism and hypothyroidism through a euthyroid state. The term "? thyroid disorder" has been applied to a few subjects who had a certain number of signs, symptoms or objective measurements indicative of hyper- or hypothyroidism, but in whom the diagnosis could not be established with reasonable certainty for one reason or another, usually because the patients were not under observation for a sufficient length of time. The majority of these subjects probably had either hypo- or hyperthyroidism. The patients with pituitary disorders, chiefly hypopituitary, have been listed separately, as have those with Addison's disease, although there is good reason to believe that most of them had secondary hypothyroidism $(11,12)$. The other categories are more or less self-explanatory. Some of the patients belonged in more than one category. For the purposes of this summary table, however, they have been listed only once. Since the most important distinction is that between thyroid disorders and other cliseases, the thyroid class has been given priority in the allocation of subjects. It follows that this class includes patients with a great variety of conditions that would otherwise warrant their allocation to another category.

In earlier analyses of lipids in thyroid disease the interrelationships of the lipid fractions which were measured proved to be normal (13). There were, however, few fractionations of cholesterol. On the basis of these few it was tentatively suggested that the ratio of free to total cholesterol also remained within normal limits. This was more a prediction than an assertion. On the basis of a larger body of data, together with published reports of measurements made by equally reliable methods (14), this prediction appears to be justified. In this series cholesterol was fractionated in 92 sera from 46 patients with disorders of the thyroid and in 16 sera from seven patients with questionable disorders. The cholesterol ratio, free: total, lay within the normal limits, 0.24 to 0.32 , in 67 sera from 36 subjects with disorders of the thyroid and in nine sera from four patients with questionable disorders of the thyroid. It was above the normal limits in 26 sera from $13^{2}$ patients with disorders of the thyroid and in seven sera from three patients with questionable disorders. The fact that 26 out of 92 ratios, or almost a third, were abnormally high seems statistically important. For reasons not connected with the study of thyroid disorders, however, an unusually large proportion of observations was made upon patients who, on other accounts, could be

2 Two patients had at different times normal and elevated ratios. 
expected to have abnormal ratios. Of the patients who had abnormal ratios, five had diseases of the liver or bile ducts (cirrhosis, infectious hepatitis or biliary obstruction); four had diabetes, complicated respectively by pulmonary tuberculosis, intercapillary glomerulosclerosis, pyelonephritis, and cellulitis of the foot with possible cirrhosis; one had disseminated tuberculosis; one had myxedema and lobar pneumonia which proved fatal on the following day. All these conditions could be associated with abnormal cholesterol ratios in patients without thyroid disorders. The remaining five belong to a group which has been described elsewhere (14) who developed late febrile reactions with rheumatic manifestations during treatment with thio-drugs. All these patients had some eviclence of impaired liver function manifested in liver function tests. In only one of these did the ratio exceed 0.34 at any time. This patient had definite hepatic disease associated with jaundice. Of the 33 abnormal ratios only 16 exceeded 0.35 . Total cholesterol of patients with normal ratios varied from 104 to $911 \mathrm{mg}$. per cent; SPI varied from 0 to $22.5 \gamma$ per cent. It seems justifiable, therefore, to conclude that a ratio of free to total cholesterol exceeding 0.32 in the serum of a patient with hyper- or hypothyroidism must be attributed to the presence of a complicating condition.

The serum of normal persons in the postabsorptive state contains little neutral fat. The fatty acids of the serum belong chiefly to phospholipids and cholesterol esters. In the previous report (13) it was stated that the concentration of neutral fat did not appear to be affected by disorders of thyroid function. In the analysis on which this statement was based, however, cholesterol was not regularly fractionated. Instead it was assumed that the ratio of free to total cholesterol in the sera of all subjects was 0.28 , the mean ratio in normal sera. In the present series complete fractionation of lipids, permitting the estimation of neutral fat, was carried out on 81 sera from 46 patients with unquestionable and five patients with questionable disorders of thyroid function. In 47 observations on 30 patients with definite and four with questionable disorders the fatty acid of neutral fat was less than $6.0 \mathrm{mM}$. per liter. This figure represents the normal mean plus twice the standard deviation (15). The concentration of neutral fat-fatty acid exceeded $6.0 \mathrm{mM}$. per liter 34 times in 18 patients with definite and one with questionable disorders of the thyroid. Of these patients four had diabetes with complications, two had renal disease, four had hepatic disease and one had an intracranial lesion-probably a tumor in the region of the pituitary. Increases of neutral fat in the serum have been reported in all these conditions (1619). Four of the remaining seven belonged to the group of patients who developed fever and arthritic symptoms during treatment with thio-drugs. One had disseminated tuberculosis as well. The presence of high serum fat in these subjects and its possible relation to impairment of liver function have been mentioned elsewhere (20). Another presented a most peculiar picture. She had suffered for many years from tachycardia and peculiar attacks of dyspnea and precordial pain, evidently precipitated by emotional factors, by which she had become completely invalided. Nevertheless she had become so grossly obese and slightly edematous as well, that physical examination was almost useless. Although both basal metabolism and SPI were elevated and the latter was reduced to normal by thio-drugs, her symptoms were not appreciably relieved by the therapy. It is generally believed that she has some condition other than hyperthyroidism, the nature of which has never been elucidated. This leaves four hypothyroid patients without obvious complications with excessive neutral fat-fatty acids. In two with moderately elevated total cholesterol the concentrations of these fatty acids were only slightly elevated, 7.7 and $11.2 \mathrm{mM}$. per liter. A third with outspoken myxedema, a serum cholesterol of $688 \mathrm{mg}$. per cent and xanthomatosis tuberosa had $16.9 \mathrm{mM}$. of neutral fat-fatty acids. The fourth, with a cholesterol of $911 \mathrm{mg}$. per cent, had $20.9 \mathrm{mM}$. of neutral fat-fatty acid. This might suggest that neutral fat rises with the serum lipids as a whole. There is, however, no evidence of such a relation. Among the patients with normal neutral fat, cholesterol varied from 90 to $877 \mathrm{mg}$. per cent and SPI from 0 to $19.4 \gamma$ per cent. Increases of neutral fat, therefore, rarely occur in uncomplicated disorders of the thyroid. If found in such conditions they usually betoken the presence of a complication.

In Figure 1 the concentration of SPI is compared with that of cholesterol. The solid circles 


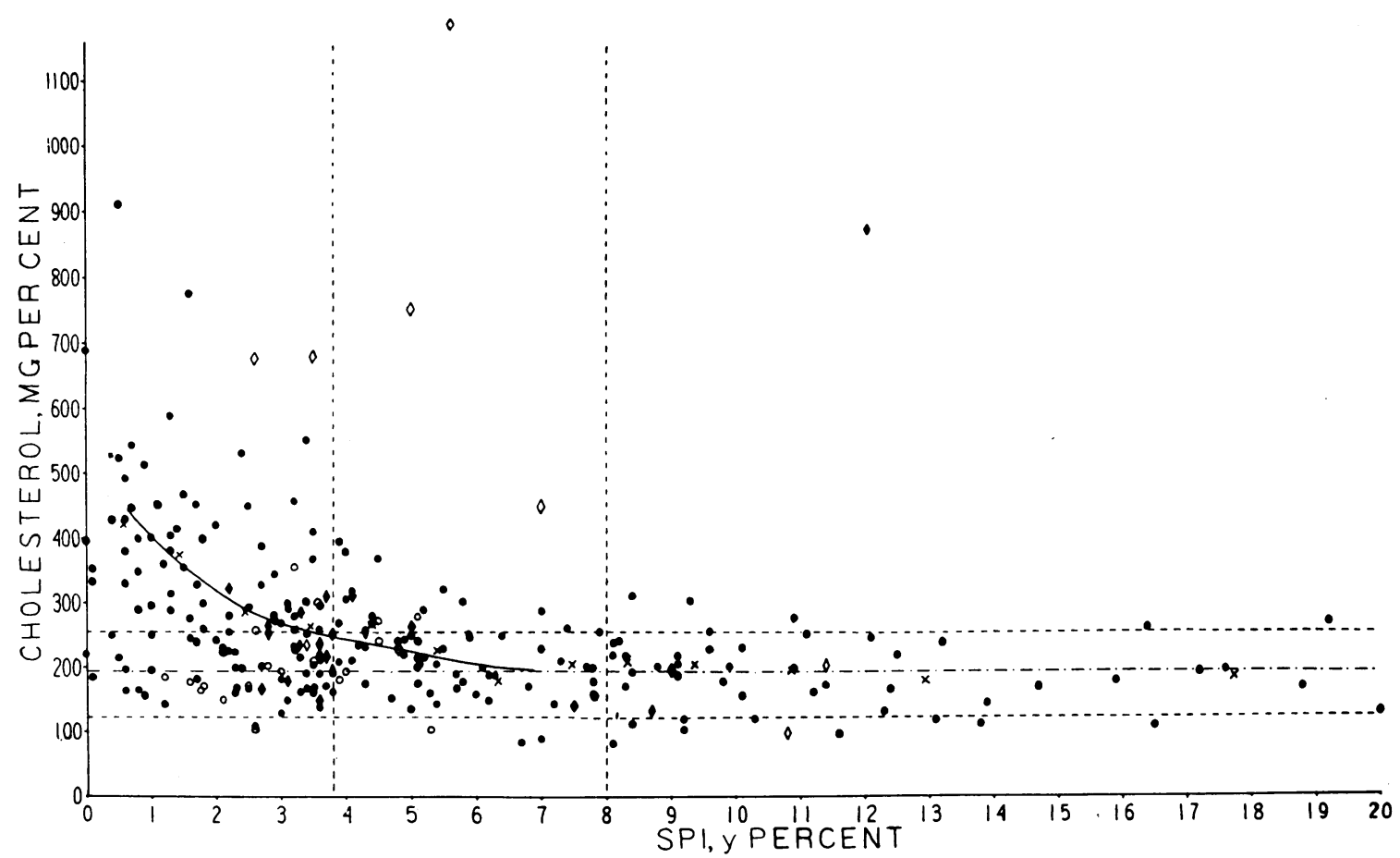

Fig. 1. The Relation of Serum Precipitable Iodine to Serum Cholesterol. in Patients with Disorders of Thyroin Function

The vertical broken lines define the limits of normal variation of SPI. The dash-dot horizontal line indicates the mean concentration of cholesterol in normal serum. The broken horizontal lines define an area including twice the standard deviation from the mean. Solid circles represent observations from patients with indubitable thyroid disorders; solid lozenges represent "? thyroid" cases; open circles are from patients with diseases of the pituitary; open lozenges are observations from one peculiar case described in the text. Crosses indicate the mean concentrations of cholesterol and SPI at various concentrations of SPI. The solid line is drawn to approximate the distribution of these means.

represent observations made on patients with definite disorders of the thyroid. The subjects were, in most instances, in either a hypo- or a hyperthyroid state; but a certain number of the observations were made when they were in a transitional euthyroid phase or had been rendered euthyroid by treatment. The points, therefore. in a sense delineate the relations of cholesterol and SPI of serum in the course of thyroid disorders or with varying degrees of thyroid activity. A few figures have been omitted because of their extreme divergence. These were from two patients who developed preposterously high SPI's while receiving thio-drugs (20). Observations from patients with "? hyperthyroidism" are represented by solid lozenges; those from pituitary patients by open circles. The open lozenges mark observations from. a patient with hypothyroidism, diabetes and renal disease under various conditions. The data have been distinguished from the others because the complications evidently were the chief determinants of the unusual values for serum cholesterol. The area enclosed by the vertical broken lines defines the limits of variation of SPI in normal individuals. The dash-clot horizontal line is the mean normal concentration of serum cholesterol; the broken horizontal lines include twice the standard deviation from the mean (15). The central quadrangle, therefore, defines the area of normal cholesterol and SPI. The crosses mark the mean concentrations of SPI and cholesterol at various concentrations of SPI. The solid line is drawn to approximate the distribution of these means. It has no particular significance; it is introduced chiefly as a visual aid.

The chart is overweighted with hypothyroid subjects because the measurements were made chiefly for clinical purposes. There is extreme 
scattering of cholesterol at low values of SPI. This end of the figure differs from the other chiefly by the presence of large numbers of high cholesterol values. Low values appear at all concentrations of SPI. Above an SPI of $6 \gamma$ per cent there is no reason to believe that the distribution curve is other than a straight line. The means above $5 \gamma$ per cent do not depart significantly from the normal mean. This confirms a conclusion reached in an earlier analysis (21), that it is impossible by such methods as this to demonstrate that hyperthyroidism affects serum cholesterol. The latter is only a negative aid in the diagnosis of hyperthyroidism. High cholesterol argues against hyperthyroidism, but the diagnosis can not be made on the basis of low cholesterol. From the distribution of points at the left of the figure it can be deduced that high cholesterol favors the diagnosis of hypothyroidism, but a low cholesterol does not exclude this condition.

The subject of most concern is the explanation of low values of cholesterol when SPI is subnormal, since it is these low values that invalidate the use of cholesterol for the diagnosis of hypothyroidism. Experimentally it has been demonstrated that in both animals and man removal of the thyroid gland is followed by the appearance of hypercholesterolemia $(13,22-24)$. It has been shown that malnutrition may lower serum cholesterol (25). The hypothyroid animal appears to be particularly susceptible to the effects of malnutrition. Entenman, Chaikoff and Reichert (26) were able to prevent hypercholesterolemia by restricting the diets of dogs after thyroidectomy. Cholesterol also falls sharply after operation (27) and is reduced in infectious diseases (28-32). Presumably these reductions are manifestations of the reaction to injury. In the present series cholesterol was less than $194 \mathrm{mg}$. per cent, the mean normal value, 20 times when SPI was less than $3.9 \%$ per cent. In nine instances the patients with these low values were suffering from complicating conditions that were either associated with striking malnutrition or of such a nature that they presumably provoked the injury reaction: pernicious anemia with extreme malnutrition, pneumonia, advanced tuberculosis, diabetes with arterial disease and renal insufficiency, arteriosclerotic heart disease with heart failure. One patient had been admitted to the hospital in stupor with extreme dehydration and emaciation, which responded rapidly to administration of thyroid. Concerning four patients information is inadequate. Of the remaining six determinations, three were from cretinous children; two were made during transitory hypothyroidism following thyroidectomy; one was from a subject who developed hypothyroidism under the influence of thiourea.

Another factor which probably influences the correlation of SPI with cholesterol is the difference in the response of these two variables to therapeutic measures. This is particularly evident in certain data of Riggs and Man (33). Riggs gave to two schizophrenic patients large doses of thiouracil. When the subjects had become frankly myxedematous, they were given increasing doses of thyroid, which was withdrawn when they had become hyperthyroid. Finally the thiouracil was also stopped. Weight, pulse rate, blood pressure and SPI were determined at weekly intervals; serum cholesterol and fatty acids were measured in the postabsorptive state each time the treatment was changed. The two experiments extended over periods of 110 and 117 weeks respectively. The courses of the two subjects were almost identical. The course of one subject is illustrated in abstract form in Figure 2. The mean basal metabolism before treatment was -10 per cent, cholesterol about $200 \mathrm{mg}$. per cent and SPI about $5 \gamma$ per cent. After the preliminary control period the patient was given $0.6 \mathrm{gm}$. of thiouracil daily. In the descent to myxedema the response of SPI was more prompt than that of basal metabolism. Otherwise these two functions paralleled one another closely throughout the experiment. The basal gradually fell to -35 per cent, SPI to $0.5 \gamma$ per cent, at which time cholesterol had risen to $306 \mathrm{mg}$. per cent. Possibly cholesterol would have risen further if the treatment had been continued unchanged. It had, however, remained stable for six weeks. The patient was then given $0.06 \mathrm{gm}$. of thyroid daily. At the next observation, after another six weeks. basal metabolism and SPI had risen slightly, but were still at hypothyroid levels. Cholesterol, on the other hand, had fallen to $151 \mathrm{mg}$. per cent, lower than the initial euthyroid figure. The other subject reacted in precisely the same manner. At this point, then, cholesterol misrepresented the state of thyroid activity, responding excessively 


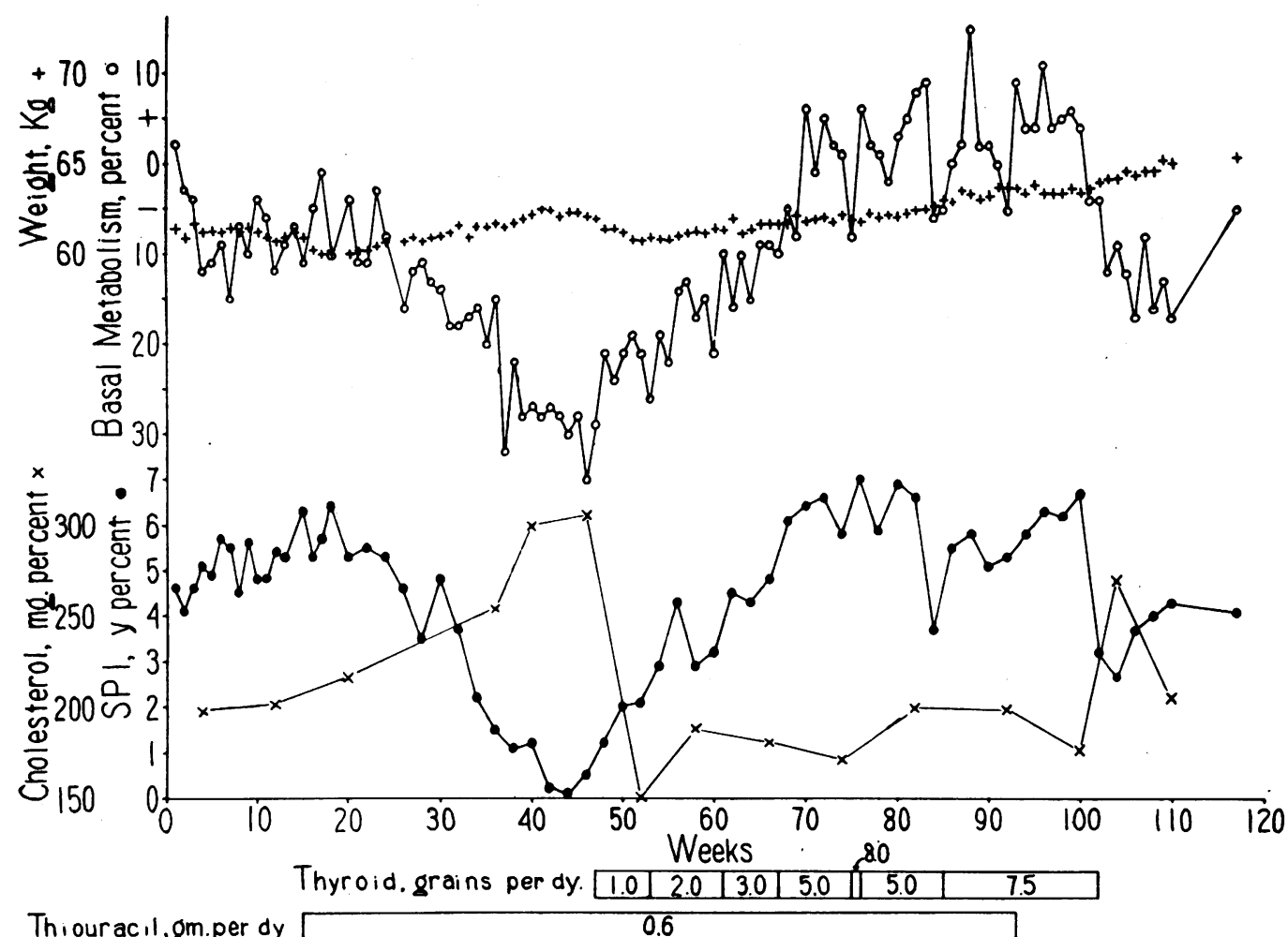

Fig. 2. The Course of Serum Precipitable Iodine and Serum Cholesterol in a Presumably Euthyroid Schizophrenic Subject under the Influence of Thiouracil and Thyroid

to an inadequate dose of thyroicl. This was not a transient reaction; the interval between these observations was six weeks. Moreover, in the following six weeks cholesterol paradoxically rose with basal metabolism and SPI. It finally reached its original value when basal metabolism and SPI had overshot their initial marks and indicated a state of hyperthyroidism. This was a temporary state, associated with no overt symptoms nor signs of excessive thyroid activity. Both basal metabolism and SPI returned to their original levels again while the subject was still receiving $0.45 \mathrm{gm}$. of thyroid, an illustration of the ability of the person with an inherently normal thyroid gland to dispose of thyroid hormone. During this period, while basal metabolism and SPI were varying, cholesterol remained relatively constant. When thyroid was discontinued, cholesterol rose above normal, varying inversely as basal metabolism and SPI, just as it had at the onset when thiouracil was given. When thiouracil was also discontinued, basal metabolism, SPI and cholesterol all returned to their original levels. The study brings out several points. It confirms the impression that, although cholesterol rises in hypothyroidism, it is not necessarily depressed by excessive thyroid activity. It also fails to mirror the course of thyroid activity as basal metabolism and SPI do. On both occasions when the subject went into frank hypothyroidism under the influence of thiouracil, cholesterol rose to abnormally high concentrations; but it fell sharply after a dose of thyroid that was too small to restore thyroid activity to normal.

This inconsistency of the response of cholesterol affords a possible explanation for a certain proportion of the low serum cholesterols observed in hypothyroidism, especially those encountered during transitory postoperative hypothyroidism and partially treated hypothyroidism. The underlying cause of the inconsistency is not clear. It is possible that it may be related to the peculiar effect of the thyroid upon the dietary or nutritive response of cholesterol. It has been repeatedly demonstrated that the serum cholesterol of normal subjects is not appreciably influenced by diet so long 
as a high level of nutrition is maintained. It appears to fall in states of malnutrition, but does not rise when excessive amounts of food are given, nor is it elevated in obesity (25). Entenman, Chaikoff and Reichert (26) found, however, that the cholesterol of thyroidectomized animals is susceptible to the effect of diet. The hypercholesterolemia of myxedema can be prevented by dietary restriction. The hypercholesterolemia produced by administration of cholesterol to rabbits is exaggerated by thyroidectomy (34-36). Other species of animals, whose serum cholesterol is not ordinarily affected by feeding cholesterol are rendered susceptible to its effects by suppression of thyroid activity (37). The initial rise of cholesterol in Figure 2 was associated with an increase of weight amounting to $9 \mathrm{lbs}$; the drop of cholesterol after $0.06 \mathrm{gm}$. of thyroid was attended by a loss of $7 \mathrm{lbs}$. These changes of weight may have been wholly or partly referable to retention and discharge of water and salt. They were, however, quite gradual and the subject had no evident edema when the weight had reached its peak. Failure of the serum cholesterol to rise in succeeding periods when her weight increased $20 \mathrm{lbs}$. is not relevant to the problem because at this time she was no longer in a hypothyroid state. It should be again emphasized that the weight curve of the second subject followed an identical course. Since the interrelationships of the lipid components are not altered by variations of thyroid activity, it follows that all the lipid fractions except neutral fat are equally affected. The disorder of hypothyroidism is not merely a hypercholesterolemia, but a hyperlipemia.

It has been difficult in the past to demonstrate gradations of thyroid insufficiency. Means (38), indeed, at one time stated that it was impossible to recognize partial insufficiency if such a condition existed. We were forced to a similar conclusion by studies of the therapeutic response to thyroid $(39,40)$. Such gradations are, however, evident in Figures 1 and 2 during the development and recovery from myxedema. The therapeutic use of thio-drugs and measurements of SPI have made it possible to recognize varying degrees of thyroid activity and have compelled a modification of the all-or-nothing view. In Figure 1 cholesterol begins to rise, not when SPI is $3.9 \gamma$ per cent, the point that marks the appearance of clini- cal hypothyroidism, but when it is as high as $6 y$ per cent. This suggests that among persons with SPI as high as this a certain proportion may have minor degrees of thyroid deficiency. The occasional incidence of hypercholesterolemia at high concentrations of SPI can be attributed to complications. It only serves to emphasize the fact that hyperthyroidism does not have a potent influence upon serum cholesterol.

The values from the "? thyroid" group in Figure 1 follow the distribution of the thyroid sub)jects. This is natural since most of those termed "? thyroid" probably belonged in the thyroid group. In the patients with pituitary disorders, low SPI is seldom accompanied by high cholesterol. These patients have been cliscussed in a separate communication (11). Finally, there are a number of points from a single patient who had hypothyroidism complicated by diabetes and, for a time, by nephritis. During her treatment she went into a temporary phase of hyperthyroidism. The extremely high cholesterol, persisting after SPI became normal, must be attributed to these complications. The hyperlipemia differed from that of hypothyroidism in that the neutral fat was greatly elevated, as it is in the hyperlipemias of diabetes and nephritis.

In Figure 3 observations on patients without hyperthyroidism are presented. The figure is constructed on the same principles as Figure 1. High values for SPI are conspicuously lacking. They were encountered in only three patients. One, with values of 12.1 and $12.4 \gamma$ per cent, had advanced chronic nephritis with hypertension. The possibility of hyperthyroidism could not be absolutely excluded. She did not, however, have obvious signs of the condition and neither SPI nor any other signs or symptoms were demonstrably altered by administration of iodine. Another, with an SPI of $8.8 \gamma$ per cent had cirrhosis of the liver.

The distribution of points bears a general resemblance to that of the thyroid patients. There is the same tendency for high cholesterols to appear more frequently when SPI is low. Among thyroid cases, however, cholesterol never exceeded $400 \mathrm{mg}$. per cent unless SPI was below $4 \gamma$ per cent; while in the non-thyroid series such high values continue up to $5 \gamma$ per cent. If these are examined all are found to come from patients with the nephrotic syndrome. Attention was 


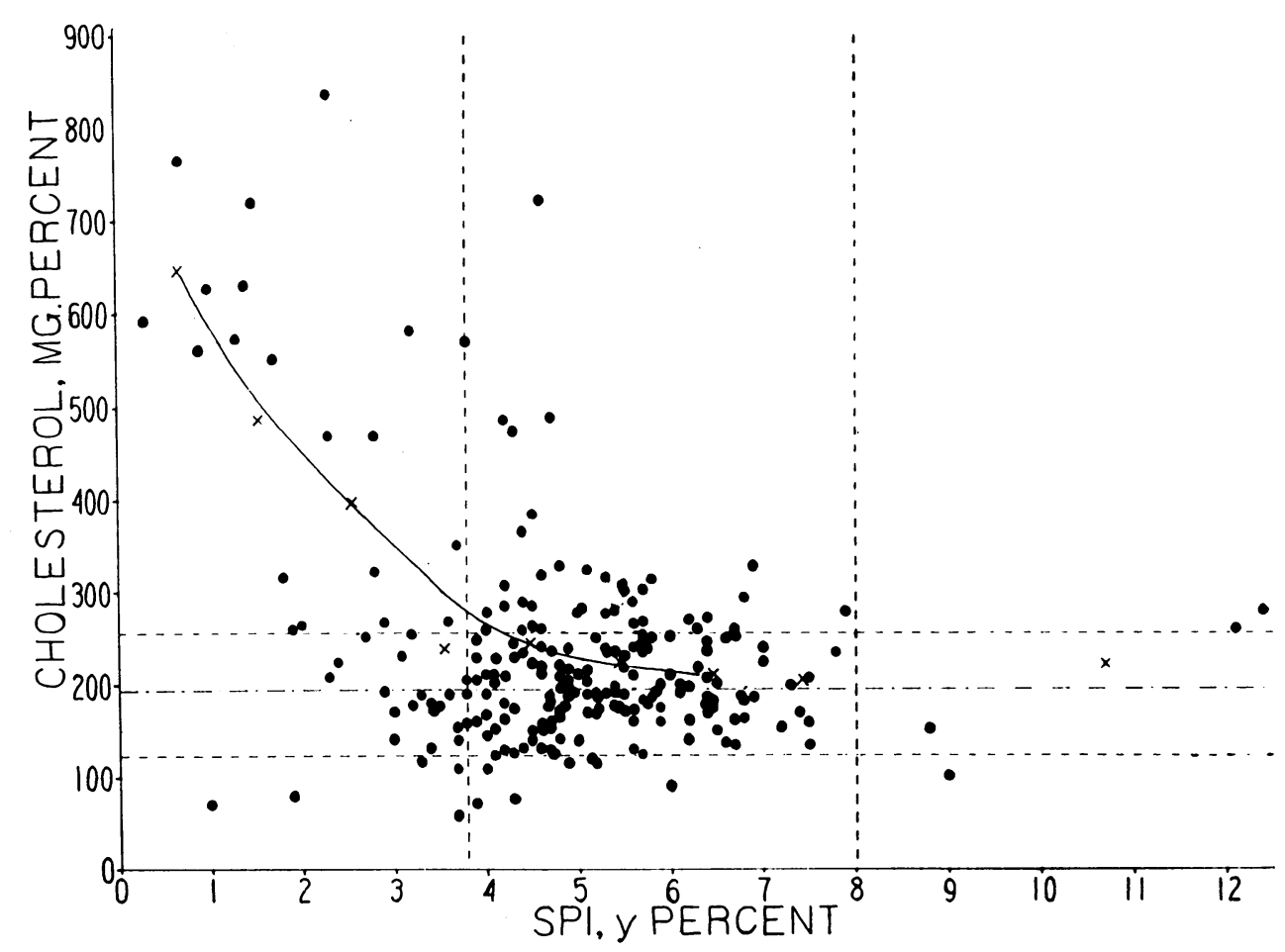

Fig. 3. The Relation of Serum Precipitable Iodine to Serum Cholesterol in Patients without Disorders of Thyroid Function

The vertical broken lines define the limits of normal variation of SPI. The dash-dot horizontal line indicates the mean concentration of cholesterol in normal serum. The broken horizontal lines define an area including twice the standard deviation from the mean. The circles represent observations from patients without thyroid disorders. Crosses indicate the mean concentrations of cholesterol and SPI at various concentrations of SPI. The solid line is drawn to approximate the distribution of these means.

drawn to this in an earlier paper (41) in which it was suggested that the low SPI was related to the low concentration of serum albumin in these subjects. The combination of low albumin with low SPI was found in sera of a few patients without nephritis or a nephrotic syndrome. Further investigation of the subject has thrown some doubt on this explanation. Extreme reductions of serum albumin are usually accompanied by low SPI ; but no correlation can be discovered between albumin on the whole and SPI. Moreover, in certain diseases, low SPI occurs while serum albumin is normal or only slightly reduced. The conjunction of low SPI with low albumin may be a mere coincidence dependent upon the fact that serum albumin deficits are common in those disorders other than hypothyroidism in which SPI is reduced.

Of the 16 points with SPI less than $4.0 \gamma$ per cent and cholesterol greater than $300 \mathrm{mg}$. per cent,
14 belong to two patients with outspoken nephrotic syndromes, one to a patient with diabetes and intercapillary nephrosclerosis. No diagnosis was established on the remaining patient. Symptoms of anorexia, diarrhea and general osteoporosis suggest that she had steatorrhea, but this was not investigated. In addition, she had a hemangioma of the skull. Of the six patients with SPI below $4.0 \gamma$ per cent and cholesterol below $123 \mathrm{mg}$. per cent, three had cirrhosis of the liver, one had constrictive pericarditis, one had exfoliative dermatitis, and the last was in the terminal stage of intercapillary nephrosclerosis. Of those with low SPI, but normal cholesterol, two had cirrhosis of the liver, one carcinoma of the pancreas with biliary obstruction, a fourth had constrictive pericarditis with extreme enlargement of the liver and intractable ascites. These patients can, therefore, be placed in the general category of liver disease. Two had the nephrotic syndrome and three had 
nephritis. One had diabetes, rheumatic heart disease and severe heart failure, and another was in the terminal stage of pulmonary tuberculosis. Three had Addison's disease with goiters and symptoms and signs suggesting hypothyroidism. Another had hemochromatosis with destruction of the adrenal glands. These cases also will be discussed in a separate communication (12). The remaining 10 subjects were suffering from various types of psychoses. This excludes the psychopathic patients who had other evidences of hypothyroidism. The frequency of low SPI in psychopathic patients deserves further inquiry. The features which in other cases appear to be responsible for serum iodine deficits without thyroid disorders, were not noted in members of this series. It is, however, difficult to evaluate symptoms and signs of persons in highly disordered states. Low SPI did not appear to he associated with any particular type of mental disorder. It could not be correlated with the state of nutrition. It may be significant that cholesterol in all these cases was within normal limits.

\section{DISCUSSION}

It is evident from this analysis that serum cholesterol is of limited value in the diagnosis or the control of treatment of thyroid disorders. It would appear from random observations that overactivity of the thyroid gland has no effect on serum cholesterol. That this is not precisely true was pointed out in a previous paper. When hyperthyroidism is benefited by administration of iodine, cholesterol usually rises perceptibly (21). These rises are, however, not consistent; they are extremely variable and not correlated with the changes of basal metabolism or other evidences of improvement. For the most part the serum cholesterols of patients with uncomplicated hyperthyroidism remain within normal limits. These limits are so wide that the effect of hyperthyroidism can be detected only by the response to therapy. How capricious this response may be is evident from Figure 2. Outspoken hypothyroidism does cause serum cholesterol to rise to a variable degree. In this rise both fractions of cholesterol share proportionally. Phospholipids also increase proportionally. Neutral fat, on the other hand, does not appear to rise. When hypothyroidism is modified by therapy cholesterol responds more rapidly than other criteria of thyroid activity. It may return to normal before basal metabolism and SPI have been appreciably affected. With degrees of hypothyroidism intermediate between outspoken myxedema and euthyroidism concentrations of cholesterol in the serum appear to be quite capricious. In addition, cholesterol is influenced by nutritive deficiencies and complicating disorders that do not affect SPI comparably. In hypopituitary states low SPI is usually accompanied by normal serum cholesterol although the iodine deficiency is probably referable to hypothyroidism (11). A similar lack of correlation is found in a series of patients with Addison's disease and low SPI (12). It may be that the failure of cholesterol to rise in these cases is directly attributable to deficient anterior pituitary or adrenocortical activity. The experiments of Entenman, Chaikoff and Reichert (26) would rather suggest that it was more closely related to secondary consequences of these glandular deficiencies. It may only signify that the activity of the thyroid is not completely suppressed in these conditions. In any case the clinical implications are clear: serum cholesterol is of little or no value in the detection of hypothyroidism secondary to pituitary or adrenal insufficiency.

The relations between SPI and serum cholesterol in patients with and those without thyroid disorders appear strangely similar. In both, cholesterol appears to be indifferent to variations of SPI in the upper part of or above the normal range. When SPI falls below these concentrations cholesterol is frequently elevated. When, however, these high cholesterol values are examined, it is found that all but one were derived from patients with the nephrotic syndrome. Because, in the nephrotic syndrome with serum albumin deficiency, hypercholesterolemia is associated with low basal metabolism, Epstein and Lande $(42,43)$ concluded that thyroid activity in this disorder was reduced and advocated the therapeutic use of thyroid. The discovery that SPI is also low in this condition seems to lend plausibility to this view. Clinical signs of hypothyroidism, however, are lacking and the patients do not respond as hypothyroid patients do to administration of thyroid. The hyperlipemia of nephrosis is also distinguished from that of hypothyroidism by the fact that neutral fat is greatly increased in the 
former (18). The relation of SPI to serum cholesterol also differs in some respects in the two conditions. In hypothyroidism the concentration of cholesterol in the serum seldom rises to great heights unless thyroid function and SPI are reduced to a minimum, and responds more rapidly than the latter to treatment. In nephrosis, on the other hand, cholesterol may be greatly elevated while SPI is still in the normal range, and is peculiarly resistant to thyroid therapy. A similar inconsistency between cholesterol and basal metabolism in nephrosis has been reported. The reduction of SPI is not, as was first supposed (41), correlated with serum albumin deficits, although SPI is usually low in the presence of extreme albumin deficits. Values lower than $3.8 \gamma$ per cent have been observed in patients with nephritis who have only slight serum albumin deficits.

Low SPI without hypothyroidism has also been encountered in patients with advanced destruction or degeneration of the liver, especially in the late stages of cirrhosis. In these conditions serum cholesterol is normal or subnormal, and the ratio, free : total cholesterol, is usually elevated. A number of patients with advanced heart failure, tuberculosis and other debilitating conditions have had low SPI with normal or reduced cholesterol, without signs or symptoms of thyroid deficiency. The incidence and reasons for these SPI deficits will be discussed elsewhere (44). The failure of cholesterol to rise in these cases can not be used as evidence that thyroid activity is not diminished because in similar conditions without hypothyroidism and with normal SPI cholesterol may be reduced. The frequency of low SPI in patients with psychopathic disorders far exceeds any reasonable expectancy. From the data available these could not be correlated with the nutritive state or any complicating factors. In a certain proportion, basal metabolism was measured and was usually reduced to a variable degree, but cholesterol was not elevated. The subject requires more investigation with therapeutic tests.

\section{CONCLUSIONS}

Serum precipitable iodine and cholesterol have been compared in large series of patients with and without disorders of thyroid function. Cholesterol is of no aid in the detection of overactivity of the thyroid. Although it rises in outspoken uncomplicated hypothyroidism, its concentration is not correlated with the degree of thyroid deficiency. Reasons for this lack of correlation are discussed.

\section{BIBLIOGRAPHY}

1. Taurog, A., and Chaikoff, I. L., The nature of the circulating thyroid hormone. J. Biol. Chem., 1948, 176, 639.

2. Man, E. B., Smirnow, A. E., Gildea, E. F., and Peters, J. P., Serum iodine fractions in hyperthyroidism. J. Clin. Invest., 1942, 21, 773.

3. Man, E. B., and Gildea, E. F., A modification of the Stoddard and Drury titrimetric method for the determination of the fatty acids in blood serum. J. Biol. Chem., 1932, 99, 43.

4. Man, E. B., and Gildea, E. F., Notes on the extraction and saponification of lipids from blood and blood serum. J. Biol. Chem., 1937, $122,77$.

5. Man, E. B., and Peters, J. P., Gravimetric determination of serum cholesterol adapted to the Man and Gildea fatty acid method, with a note on the estimation of lipoid phosphorus. J. Biol. Chem., 1933, $101,685$.

6. Man, E. B., A note on the stability and quantitative determination of phosphatides. J. Biol. Chem., 1937, 117, 183.

7. Bogdanovitch, S. B., and Man, E. B., The effects of castration, theelin, testosterone and antuitrin-S on the lipoids of blood, liver and muscle of guinea pigs. Am. J. Physiol., 1938, 122, 73.

8. Howe, P. E., The determination of proteins in blood -a micro method. J. Biol. Chem., 1921, 49, 109.

9. Milne, J., Serum protein fractionation : a comparison of sodium sulfate precipitation and electrophoresis. J. Biol. Chem., 1947, 169, 595.

10. Majoor, C. L. H., The possibility of detecting individual proteins in blood serum by differentiation of solubility curves in concentrated sodium sulfate solutions. II. Comparison of solubility curves with results of electrophoresis experiments. J. Biol. Chem., 1947, 169, 583.

11. Peters, J. P., German, W. J., and Man, E. B., Serum precipitable iodine in patients with tumors of or near the pituitary. J. Clin. Endocrinol., in press.

12. Kydd, D. M., Man, E. B., and associates, Serum precipitable iodine in hypoadrenalism. To appear.

13. Peters, J. P., and Man, E. B., The interrelations of serum lipids in patients with thyroid disease. $\mathrm{J}$. Clin. Invest., 1943, 22, 715.

14. McElroy, J. S., Schuman, E. B., and Ritchey, J. O., A study of the changes in serum cholesterol, gastric secretion and carbohydrate metabolism in patients with toxic goiter. Ann. Int. Med., 1938, 12, 106.

15. Peters, J. P., and Man, E. B., The interrelations of serum lipids in normal persons. J. Clin. Invest., 1943, $22,707$. 
16. Man, E. B., and Peters, J. P., Lipoids of serum in diabetic acidosis. J. Clin. Invest., 1934, 13, 237.

17. Man, E. B., and Peters, J. P., Serum lipoids in diabetes. J. Clin. Invest., 1935, 14, 579.

18. Peters, J. P., and Man, E. B., The interrelations of serum lipids in patients with diseases of the kidneys. J. Clin. Invest., 1943, 22, 721.

19. Man, E. B., Kartin, B. L., Durlacher, S. H., and Peters, J. P., The lipids of serum and liver in patients with hepatic diseases. J. Clin. Invest., 1945, 24, 623.

20. Peters, J. P., Man, E. B., Kydd, D. M., Engstrom, W. W., and Waters, L. L., Toxic effects of antithyroid drugs. Yale J. Biol. Med., in press.

21. Man, E. B., Gildea, E. F., and Peters, J. P., Serum lipoids and proteins in hyperthyroidism. J. Clin. Invest., 1940, 19, 43.

22. Fleischmann, W., Shumacher, H. B., Jr., and Wilkins, L., The effect of thyroidectomy on serum cholesterol and basal metabolic rate in the rabbit. Am. J. Physiol., 1940, 131, 317.

23. Gilligan, D. R., Volk, M. C., Davis, D., and Blumgart, H. L., Therapeutic effect of total ablation of normal thyroid on congestive heart failure and angina pectoris. VIII. Relationship between serum cholesterol values, basal metabolic rate and clinical aspects of hypothyroidism. Arch. Int. Med., 1934, 54, 746.

24. Thompson, K. W., and Long, C. N. H., The effect of hypophysectomy upon hypercholesterolemia of dogs. Endocrinology, 1941, 28, 715.

25. Man, E. B., and Gildea, E. F., Serum lipoids in malnutrition. J. Clin. Invest., 1936, 15, 203.

26. Entenman, C., Chaikoff, I. L., and Reichert, F. L., Blood lipids of the hypophysectomized-thyroidectomized dog. Endocrinology, 1942, 30, 802.

27. Man, E. B., Bettcher, P. G., Cameron, C. M., and Peters, J. P., Plasma $\alpha$-amino acid nitrogen and serum lipids of surgical patients. J. Clin. Invest., 1946, 25, 701.

28. Denis, W., Cholesterol in human blood under pathological conditions. J. Biol. Chem., 1917, 29, 93.

29. Kipp, H. A., Variation in the cholesterol content of the serum in pneumonia. J. Biol. Chem., 1920, 44, 215.

30. Marino, S., La lipemia nelle malattie infettive acute. Arch. di farmacol. sper., 1933, 55, 1.
31. Rosen, I., Krasnow, F., and Lyons, M. A., Cholesterol and lecithin studies in syphilis. Cholesterol partition in relation to the Wassermann reaction. Arch. Dermatol. \& Syph., 1933, 27, 383.

32. Stoesser, A. V., and McQuarrie, I., Influence of acute infection and artificial fever on the plasma lipids. Am. J. Dis. Child., 1935, 49, 658.

33. Riggs, D. S., Man, E. B., and associates, Experimental hypothyroidism. Unpublished.

34. Turner, K. B., Studies on the prevention of cholesterol atherosclerosis in rabbits. I. The effects of whole thyroid and of potassium iodide. J. Exper. Med., 1933, 58, 115.

35. Turner, K. B., and Bidwell, E. H., Further observations on the blood cholesterol of rabbits in relation to atherosclerosis. J. Exper. Med., 1935, 62, 721.

36. Turner, K. B., Present, C. H., and Bidwell, E. H., The role of the thyroid in the regulation of the blood cholesterol of rabbits. J. Exper. Med., 1938, 67, 111.

37. Steiner, A., and Kendall, F. E., Atherosclerosis and arteriosclerosis in dogs following ingestion of cholesterol and thiouracil. Arch. Path., 1946, 42, 433.

38. Means, J. H., The Thyroid and Its Diseases. J. B. Lippincott Company, Philadelphia, 1937.

39. Winkler, A. W., Criscuolo, J., and Lavietes, P. H., Quantitative relationship between basal metabolic rate and thyroid dosage in patients with true myxedema. J. Clin. Invest., 1943, 22, 531.

40. Winkler, A. W., Lavietes, P. H., Robbins, C. L., and Man, E. B., Tolerance to oral thyroid and reaction to intravenous thyroxine in subjects without myxedema. J. Clin. Invest., 1943, 22, 535.

41. Peters, J. P., and Man, E. B., The relation of albumin to the precipitable iodine of serum. J. Clin. Invest., 1948, 27, 397.

42. Epstein, A. A., Further observations on the nature and treatment of chronic nephrosis. Am. J. M. Sc., 1922, 163, 167.

43. Epstein, A. A., and Lande, H., Studies on blood lipoids. I. The relation of cholesterol and protein deficiency to basal metabolism. Arch. Int. Med., 1922, 30, 563.

44. Kydd, D. M., Man, E. B., and associates, Serum precipitable iodine in conditions other than thyroid disorders. To appear. 